\title{
On Switching of a Flip-Flop Jet Nozzle with Double Ports by Single-Port Control
}

\author{
Tatsuya Inoue, Fumiaki Nagahata, Katsuya Hirata \\ Department of Mechanical Engineering, Doshisha University, Kyoto, Japan \\ Email:euo1503@mail4.doshisha.ac.jp
}

How to cite this paper: Inoue, T., Nagahata, F. and Hirata, K. (2016) On Switching of a Flip-Flop Jet Nozzle with Double Ports by Single-Port Control. Journal of Flow Control, Measurement \& Visualization, 4, 143161.

http://dx.doi.org/10.4236/jfcmv.2016.44013

Received: June 17, 2016

Accepted: August 17, 2016

Published: October 10, 2016

Copyright $\odot 2016$ by authors and Scientific Research Publishing Inc. This work is licensed under the Creative Commons Attribution International License (CC BY 4.0).

http://creativecommons.org/licenses/by/4.0/

\begin{abstract}
This research deals with the oscillation mechanism of a flip-flop jet nozzle with a connecting tube, based on the measurements of pressures and velocities in the connecting tube and inside the nozzle. The measurements are carried out varying: 1) the inside diameter $d$ of the connecting tube; 2 ) the length $L$ of the connecting tube and 3 ) the jet velocity $V_{\mathrm{PN}}$ from a primary-nozzle exit. We assume that the jet switches when a time integral reaches a certain value. At first, as the time integral, we introduce the accumulated flow work of pressure, namely, the time integral of mass flux through a connecting tube into the jet-reattaching wall from the opposite jet-un-reattaching wall. Under the assumption, the trace of pressure difference between both the ends of the connecting tube is simply modeled on the basis of measurements, and the flow velocity in the connecting tube is computed as incompressible flow. Second, in order to discuss the physics of the accumulated flow work further, we conduct another experiment in single-port control where the inflow from the control port on the jet-reattaching wall is forcibly controlled and the other control port on the opposite jet-un-reattaching wall is sealed, instead of the experiment in regular jet's oscillation using the ordinary nozzle with two control ports in connection. As a result, it is found that the accumulated flow work is adequate to determine the dominant jetoscillation frequency. In the experiment in single-port control, the accumulated flow work of the inflow until the jet's switching well agrees with that in regular jet's oscillation using the ordinary nozzle.
\end{abstract}

\section{Keywords}

Flip-Flop Jet Nozzle, Flowmeter, Fluidics, Mixing, Flow Control

\section{Introduction}

The flip-flop jet nozzle (hereinafter, referred to as FFJN) is regarded as one kind of flui- 
dic oscillator, which is oscillating devices among the fluidics. The fluidics, or the elements in fluid logic, is applications of the Coanda effect where a jet reattaches to a solid side wall, and has been researched since the 1960s [1]-[5]. The FFJN retains useful features as well as other flow-induced-vibration devices: namely, 1) low production cost and high reliability due to non-mechanically-moving parts; 2) usability due to a linear frequency response in proportion to flow rate; and 3) robustness against fluid density, temperature, pressure and composition. Owing to the above features, the FFJN is applicable for such products as flow meters, fuel injectors, micro mixers and various control devices to disturb the shear layer or to enhance heat transfer, not only for singlephase flows but also for multi-phase flows. Thus, the FFJN is often called a "fluidic oscillator" or "oscillatory-jet-type flowmeter" in different applications.

In such a context, there have been many researches on the FFJN in both fundamental and practical approaches [6]-[22]. Besides, various simplified FFJN's without control ports, chambers, feedback loop (or connecting tube) and so on, have been reported [23]-[30].

Among them, in order to reveal the flow inside the FFJN, we have carried out the measurements of unsteady flow-velocity distributions by an ultrasound-velocity-profile (UVP) monitor which gives us instantaneous information with higher accuracy in comparison to the conventional particle-image velocimetry [19]. Because the flow inside the FFJN is usually turbulent with random perturbations in addition to a dominant jet's oscillation, we conducted conditional sampling by the phase-averaging technique using the flow velocity in the connecting tube as a reference signal. However, the oscillation mechanism of the FFJN has not been fully understood yet. In general, it is still difficult to predict the jet-oscillation frequency, which depends upon such various parameters as connecting-tube length, connecting-tube volume, flow rate, nozzle's geometries and so on according to Raman et al. [12], even in the present state.

Our purpose is to elucidate the oscillation mechanism of the FFJN. In the present study, we focus upon a dominant jet-oscillation frequency of the FFJN, based on the measurements of pressures and velocities in the connecting tube and inside the FFJN, and attempt to find out the universal number which determines the jet-oscillation frequency. The measurements are carried out, varying: 1) the inside diameter $d$ of the connecting tube; 2) the length $L$ of the connecting tube and 3) the jet velocity $V_{\mathrm{PN}}$ from a primary-nozzle exit. We assume that the jet switches when a time integral reaches a certain value. At first, as this time integral which can be the universal number for the jet's switching, we introduce the accumulated flow work of pressure, namely, the time integral of mass flux through a connecting tube into the jet-reattaching wall from the opposite jet-un-reattaching wall. Under the assumption, the trace of pressure difference between both the ends of the connecting tube is simply modeled on the basis of measurements. Such modeling is the same as Funaki et al. [31]. Using this model, the flow velocity in the connecting tube is computed by the Runge-Kutta method as incompressible flow. Second, in order to discuss the physics of the accumulated flow work further, we conduct another experiment in single-port control where the inflow from one con- 
trol port on the jet-reattaching wall is forcibly controlled by a blower-and-value system and the other control port on the opposite jet-un-reattaching wall is sealed by a plug, as opposed to the experiment in regular oscillation using the ordinary FFJN with two control ports in connection.

More specifically, in our previous study Funaki et al. [31], the accumulated flow work was investigated, namely the time integral of momentum flux. However, there is a possibility of predicting the jet-oscillation frequency more directly. So, we attempt to examine two kinds of integrals; namely, the time integral of momentum flux, and the time integral of mass flux.

To predict the dominant frequency is very useful and strongly needed in many practical aspects, as we have not yet established any general prediction methods as mentioned above. One of the main factors preventing the establishment is the spatial-andtemporal complexity of the flow inside the FFJN: for example, quasi-steady approaches are not suitable even for very-low dominant frequencies and the momentum-theory approaches are difficult in setting the control volume. Therefore, the present approach could be effective for a breakthrough, in addition to our previous study Funaki et al. [31].

\section{Experimental Method}

\subsection{Model: FFJN}

Figure 1 shows the present model of a FFJN, together with its main dimensions. The FFJN consists of a primary nozzle, two side walls, two control ports, two chambers and a connecting tube. The two chambers with the control ports on the side walls are linked to each other by the connecting tube, to cause regular jet oscillation. The basic dimensions of the FFJN are determined according to Viets [8]. In addition to the experiment in regular jet's oscillation using the ordinary FFJN with two control ports in connection, we conduct another experiment in single-port-control where we seal one control port by a plug and forcibly feed fluid from the other un-sealed control port.

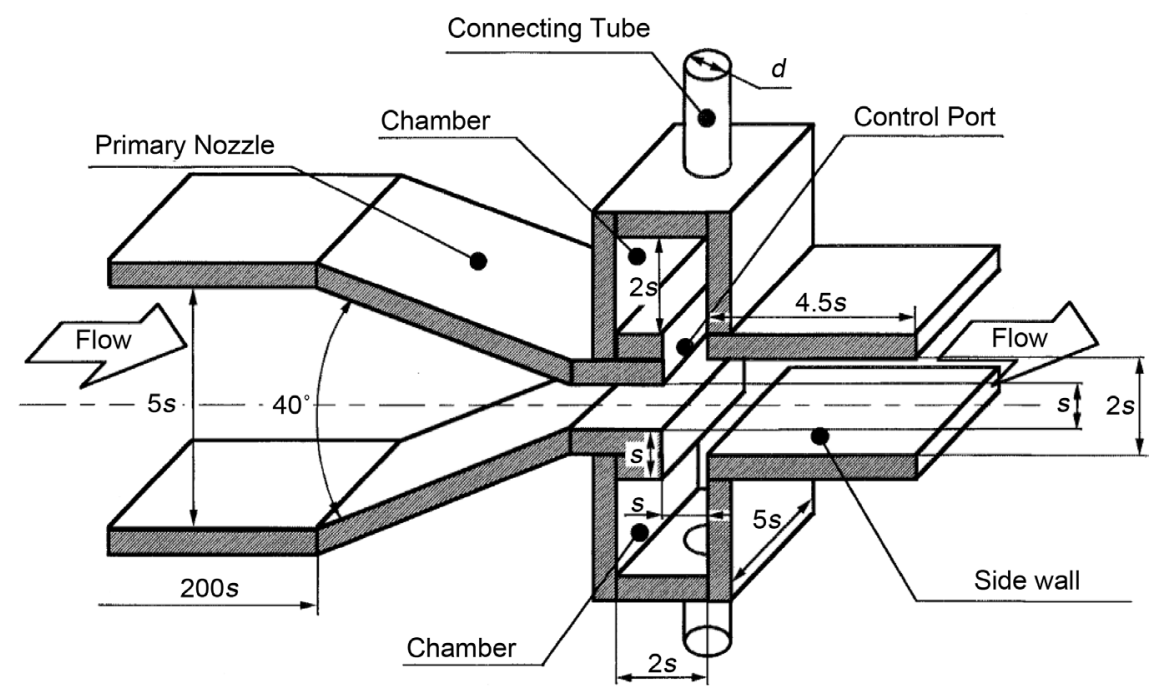

Figure 1. Model: a flip-flop jet nozzle (FFJN). 
Table 1 summarises the present experimental parameters. The chief geometric and kinetic parameters are as follows. In a characteristic length scale the spacing $s$ of the primary-nozzle throat and the nozzle span $S$ are fixed to $0.01 \mathrm{~m}$ and $0.05 \mathrm{~m}$, respectively. So, the corresponding aspect ratio $A(\equiv S / s)$ is equal to 5 . The control port with a breadth $b$ is on each side wall, where $b$ is fixed to the same as $s$ in the present study. The gap between the side walls and the streamwise length of the side walls are denoted by $G_{\mathrm{SW}}$ and $L_{\mathrm{SW}}$, respectively. Their reduced forms $G_{\mathrm{SW}} / s$ and $L_{\mathrm{SW}} / s$ are fixed to 2 and 4.5 , respectively. A sole kinetic parameter, the Reynolds number $R e$, is defined by $\rho V_{\mathrm{PN}} s / \mu$, where $\rho, V_{\mathrm{PN}}$ and $\mu$ denote the density of fluid, the mean velocity at a primary-nozzle exit and the viscosity of fluid, respectively. In the regular-oscillation experiment, the connecting tube with a length $L$ has a circular cross section with an inside diameter $d$. Their reduced forms $d / s$ and $L / s$ vary from 1.2 to 1.4 and from 100 to 300 , respectively. On the other hand, in the single-port-control experiment, a flow-increment rate coefficient $K$ (see later for its definition) varies from $1 \times 10^{-4}$ to $3.5 \times 10^{-4}$ according to the results in the regular oscillation experiment.

Table 1. Experimental parameters.

(a) Basic nozzle dimensions

\begin{tabular}{|c|c|c|c|}
\hline Primary-nozzle-throat spacing & $s$ & $(\mathrm{~m})$ & 0.01 \\
\hline Control-port spacing & $b$ & $(\mathrm{~m})$ & 0.01 \\
\hline Gap between side walls & $G_{\mathrm{SW}}$ & $(\mathrm{m})$ & 0.02 \\
\hline Streamwise length of side walls & $L_{\mathrm{SW}}$ & $(\mathrm{m})$ & 0.045 \\
\hline Span & $S$ & $(\mathrm{~m})$ & 0.05 \\
\hline Aspect ratio of primary-nozzle throat & \multicolumn{2}{|c|}{$A, \equiv S / s$} & 5 \\
\hline Reduced control-port spacing & \multicolumn{2}{|l|}{$b / s$} & 1 \\
\hline Reduced side-wall gap & \multicolumn{2}{|l|}{$G_{\mathrm{SW}} / s$} & 2 \\
\hline Reduced side-wall length & \multicolumn{2}{|l|}{$L_{\mathrm{SW}} / s$} & 4.5 \\
\hline \multicolumn{4}{|c|}{ (b) Kinetic parameter } \\
\hline Flow velocity at primary-nozzle exit & $V_{\mathrm{PN}}$ & $(\mathrm{m} / \mathrm{s})$ & $11.3-34.7$ \\
\hline Reynolds Number & $\operatorname{Re}$ & & $7500-23,000$ \\
\hline \multicolumn{4}{|c|}{ (c) Basic connecting-tube dimensions: parameters for regular oscillation } \\
\hline Connecting-tube length & $L$ & $(\mathrm{~m})$ & $1.0,1.5,2.0,2.5,3.0$ \\
\hline Connecting-tube diameter & $d$ & $(\mathrm{~m})$ & $0.012,0.013,0.014$ \\
\hline Reduced connecting-tube length & $L / s$ & & $100,150,200,250,300$ \\
\hline Reduced connecting-tube diameter & $d / s$ & & $1.2,1.3,1.4$ \\
\hline \multicolumn{4}{|c|}{ (d) Parameter for single-port control } \\
\hline Flow-increment rate & $d V_{\mathrm{T}} / d t$ & $\left(\mathrm{~m} / \mathrm{s}^{2}\right)$ & $1.8-30.7$ \\
\hline Flow-increment-rate coefficient & $K$ & & $1,1.5,2,2.5,3,3.5\left(\times 10^{-4}\right)$ \\
\hline
\end{tabular}




\subsection{Experimental Apparatus for Regular Oscillation}

Figure 2 shows the schematic diagram of the present experimental apparatus in a regular-oscillation experiment. The main part of an ordinary FFJN with a primary nozzle (No. 6 in Figure 2), two control ports, two chambers (Nos. 11 \& 12) and the connecting tube (No. 5) consists of acrylic-resin plates and a PVC tube. The working fluid is air, which is provided by an air compressor (No. 1) into the primary nozzle (No. 6) of the FFJN, through an air dryer (No. 2), a pressure regulator (No. 3), a flow meter (No. 4) and a long straight duct. The rectification duct in the upstream of the primary nozzle is straight and enough long (200s $=2 \mathrm{~m}$ ) to suppress the pulsation included in primary-nozzle jet. Volumetric flow rate into the FFJN measured by the flow meter is compensated using both the temperature and the pressure detected by a thermocouple and a pressure transducer which are placed adjacent to the flow meter. Pressures and velocities at several points are simultaneously measured by four pressure transducers KYOWA PGM-G (Nos. 7 - 10), and two hot-wire anemometers KANOMAX 7000 with I-type probes (Nos. $13 \& 14$ ), respectively. More specifically, two (Nos. $7 \& 8$ ) of the four pressure transducers are near the connecting-tube ends, one (No. 9) of the four is in the upstream of the primary nozzle, and the other (No. 10) of the four is on the sidewall inside the FFJN. One of the two hot-wire anemometers (No. 13) is for the measurement of the flow velocity inside the connecting-tube, and the other one (No. 14) is for the measurement of the flow velocity just outside the FFJN exit. The hot-wire anemometers are calibrated using a Pitot tube outside the experimental apparatus for each measurement whose duration is less than 2 hours. These signals are recorded and analyzed by a spectrum analyzer (No. 17) and a personal computer (No. 18).

\subsection{Experimental Apparatus for Single-Port Control}

Figure 3 shows the schematic diagram of the present apparatus in the single-port experiment. The FFJN has a single control port instead of dual control ports, as in the regular

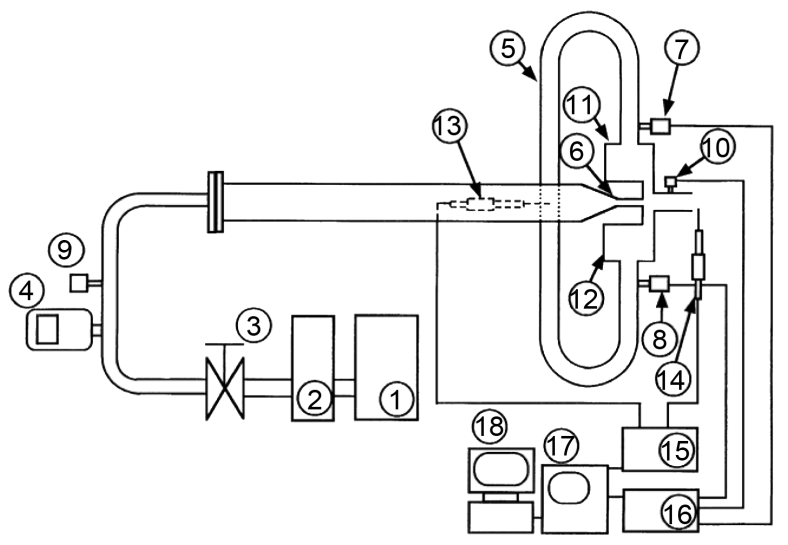

\begin{tabular}{|c|l|l|l|c|l|c|l|}
\hline (1) & Air compressor & (2) & Air dryer & (3) & Pressure regulator & (4) & Flow meter \\
\hline (5) & Connecting tube & (6) & Primary nozzle & (7)(8)(9) & Pressure transducers & (11) (12) & Chamber \\
\hline (13) & Hot-wire probe inside a connecting-tube & (14) & \multicolumn{4}{|l|}{ Hot-wire probe at a FFJN exit } \\
\hline (15) & CTA & (16) & Strain meter & (17) & Spectrum analyzer & (18) & Personal computer \\
\hline
\end{tabular}

Figure 2. Experimental apparatus for regular oscillation in ordinary FFJN operation. 


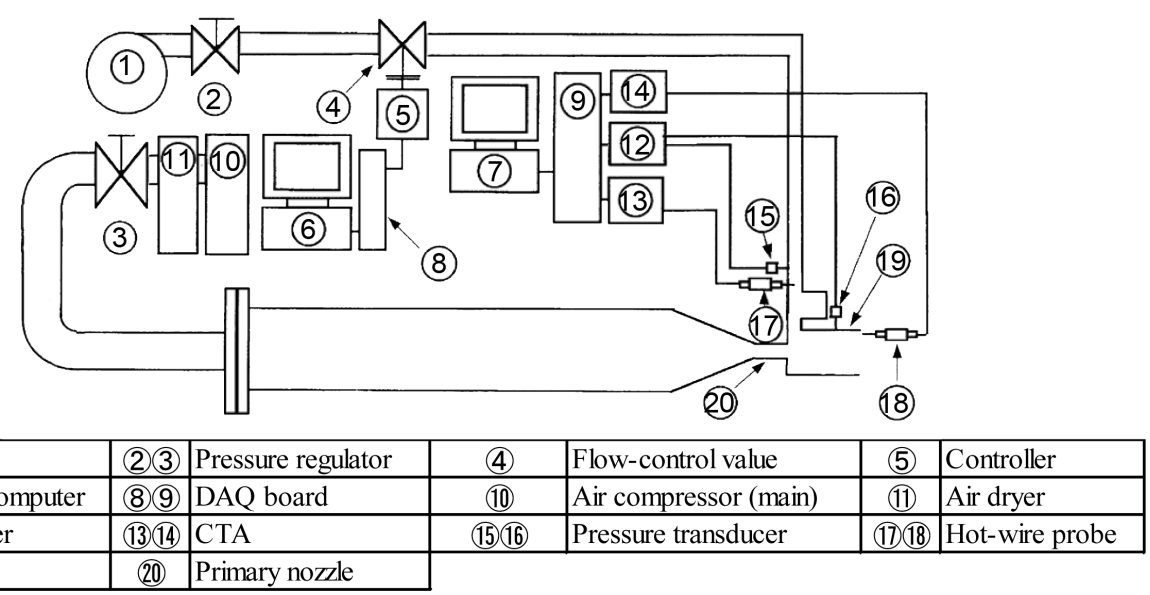

Figure 3. Experimental apparatus for single-port control.

oscillation experiment. The control port on the opposite side is sealed by a plug, being flush with a side wall. As well as the regular-oscillation experiment, the working fluid is air, which is provided by an air compressor (No. 10 in the figure) into a primary nozzle (No. 20) of the FFJN, through an air dryer (No. 11), a pressure regulator (No. 3), a flow meter and a long straight duct. Volumetric flow rate into the FFJN measured by the flow meter is compensated using both the temperature and the pressure detected by a thermocouple and a pressure transducer which are placed adjacent to the flow meter. The inflow from the control port is driven by a blower through a tube (No. 1), which is regulated by a flow-control value (No. 4). In the single-port-control experiment, the jet from the primary nozzle is reattached to the side wall with the control port in advance. Then, we force the jet to switch by the inflow, from the side wall to the opposite side wall without the control port. Pressures and velocities at several points are simultaneously measured by two pressure transducers (Nos. $15 \& 16$ ) and two hot-wire anemometers (Nos. $17 \& 18$ ), respectively. These signals are recorded and analysed by a personal computer (No. 7).

In the single-port-control experiment, we quantitatively characterise the magnitude of the inflow using volumetric flow rate $Q_{\mathrm{T}}$ from the control port through the tube, which is detected by a hot-wire anemometer at the tube end adjacent to a chamber and the control port. So, prior to the single-port-control experiment, we need the calibration between $Q_{\mathrm{T}}$ and hot-wire anemometer signal $V_{\mathrm{T}}$. Figure 4 shows the schematic diagram of the apparatus for the calibration. The working fluid is air, which is provided by a blower (No. 1 in the figure) through a tube with a diameter $d$ into a U-tube with wider cross section than the tube. Pressure and velocity at the tube end are simultaneously measured by a pressure transducer (No. 13) and a hot-wire anemometer (No. 14), respectively. The actual value of volumetric flow rate $Q_{\mathrm{T}}$ is detected by a measuring bar (No. 6) attached to a float on the anterior water surface of the U-tube. We record the value of the measuring bar by a camcorder (No. 5). These signals are recorded and analysed by a personal computer (No. 9). Figure 5 shows the result of the calibration, where the time history of the flow rate $Q_{\mathrm{T}}$ based on the flow velocity $V_{\mathrm{T}}$ is measured by 


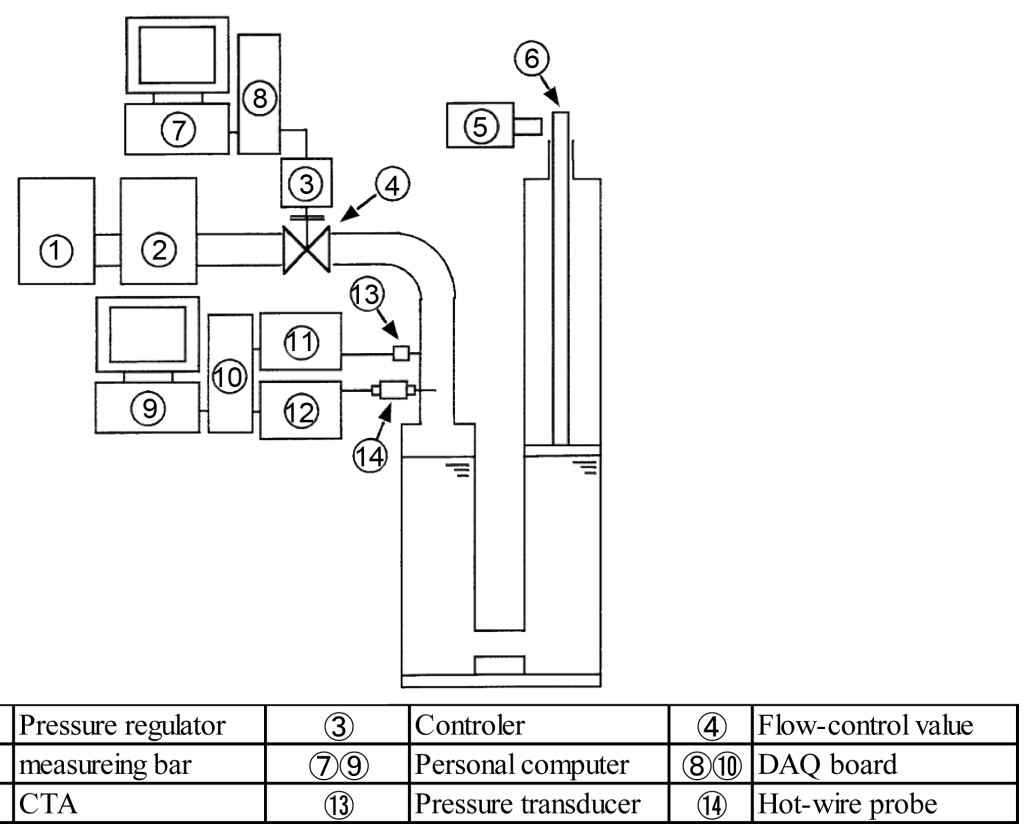

Figure 4. Experimental apparatus for the calibration of volumetric flow rate $Q_{\mathrm{T}}$.

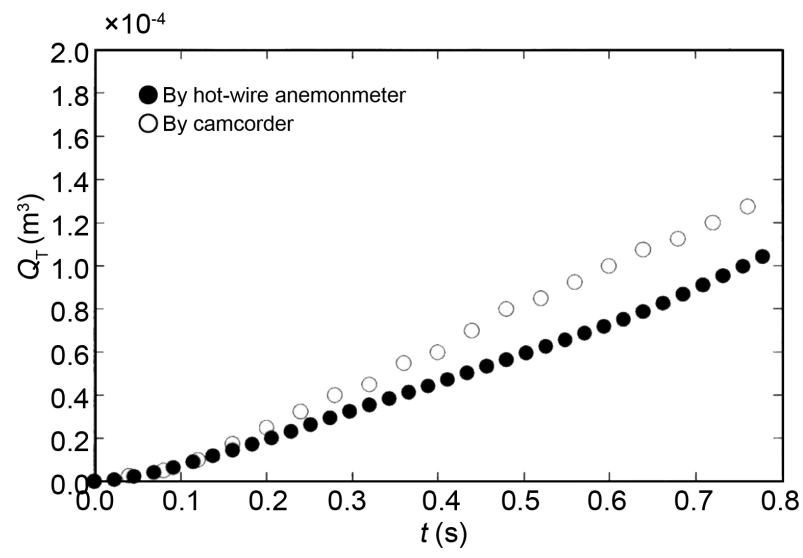

Figure 5. Flow rate $Q_{\mathrm{T}}$ based on $V_{\mathrm{T}}$ measured by a hot-wire anemometer, together with that by a camcorder.

the hot-wire anemometer, together with the actual $Q_{\mathrm{T}}$ by the hot-wire anemometer measured by a camcorder. We compensate $Q_{\mathrm{T}}$ on the basis of this result, then determine $Q_{\mathrm{T}}$ in the single-port-control experiment.

\section{Results and Discussion}

\subsection{Regular Oscillation: Jet-Oscillation Frequency and Connecting-Tube Flow}

The frequency $f$ of jet's oscillation is important not only from an academic viewpoint but also from a industrial viewpoint. According to Raman et al. [12], $f$ depends upon such various parameters as connecting-tube length, connecting-tube volume, flow rate, nozzle's geometries and so on. However, it is still difficult to predict $f$ even in the 
present stage. So, we first propose an empirical formula to determine $f$. In the present study, we get the experimental value $f$ by the Fourier analysis on the flow-velocity fluctuation detected at the FFJN exit (No. 14 in Figure 2). As governing parameters for $f$, we suppose three geometric ones in addition to mean velocity $V_{\mathrm{PN}}$ at a primary-nozzle exit, fluid density $\rho$ and fluid viscosity $\mu$. The three geometric parameters are the spacing $s$ of a primary-nozzle throat, the length $L$ of a connecting tube and the inner diameter $d$ of a connecting tube. We regard $s, V_{\mathrm{PN}}$ and $\rho$ as characteristic scales. Then, according to the dimensional analysis, we get

$$
S t=\phi(L / s, d / s, R e),
$$

where $\phi$ denotes an arbitrary function. In this Equation (1), we consider a normalised $f$, namely, the Strouhal number $S t$, instead of $f$. The definition of $S t$ is given by

$$
s t=f s / V_{\mathrm{PN}} \text {. }
$$

All the symbols in Figure 6 represent the experimental results of $S t$ plotted against $R e$ at $L / s=100-300, d / s=1.2-1.4$ and $R e=7000-20,000$. While there exist minor random scatterings, these are not due to lower stability of the present periodic flow phenomenon but due to unknown factors included in the actual experimental apparatus. From the results, we can see the following three tendencies. That is, we can see that 1) St monotonically decreases with increasing $L / s$, that 2) St monotonically increases with increasing $d / s$ and that 3) St monotonically increases with increasing $R e$. Then, we assume the following power function as $\phi$.

$$
S t=C(L / s)^{\alpha}(d / s)^{\beta} \operatorname{Re}^{\gamma},
$$

with experimental constants such as $C=0.068, \alpha=-0.72, \beta=1.37$ and $\gamma=0.22$. The experimental constants are determined using the least-squares method based on all the experimental results in Figure 6. We should note that $L / s$ is the most influential upon St among the three non-dimensional governing parameters in the present test ranges. The curves in Figure 6 show this empirical formula Equation (3). We can see that the empirical formula almost agrees with the experiment.

The empirical formula Equation (3) is practically useful not only for the present FFJN in the present test ranges of the governing parameters, but restricted due to the lack of theoretical background. Then, we consider more generally focusing upon the jet-oscillation frequency. Figure 7(a) shows a typical example of the present experiments; that is, the time history of the pressure difference $\Delta p$ between both the connecting tube ends at $L / s=100, d / s=1.2$ and $R e=13,000$. The wave form in Figure 7(a) indicates some features which are commonly seen in all the present experiments; namely, 1) close periodicity with high-frequency random fluctuations and 2) the wave form characterised by the two similar non-isosceles triangles with positive and negative signs during each jet-oscillation period. The second feature is commonly observed, whenever the wave form is almost periodic. In fact, Fourier-transform analyses on the present results always are characterised by one remarkable and stable spectrum peak representing a dominant periodicity, together with some secondary peaks representing higher harmonics due to the periodic but non-sinusoidal wave form. By means of simultaneous 


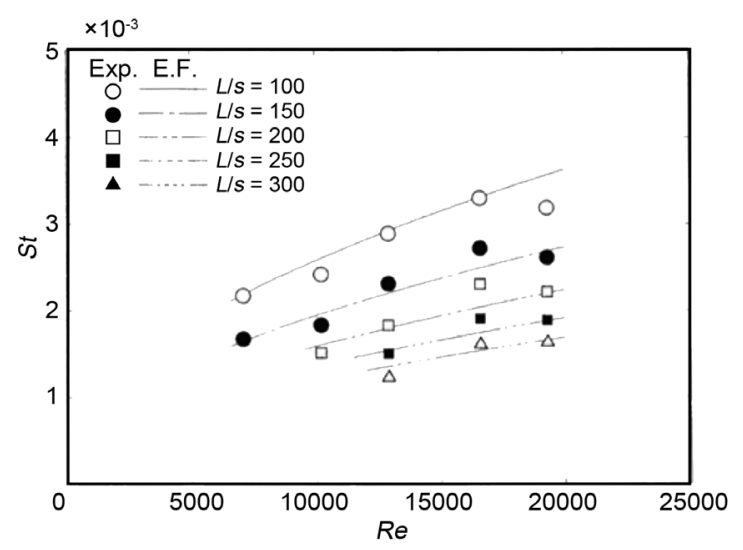

(a) $d / s=1.2$

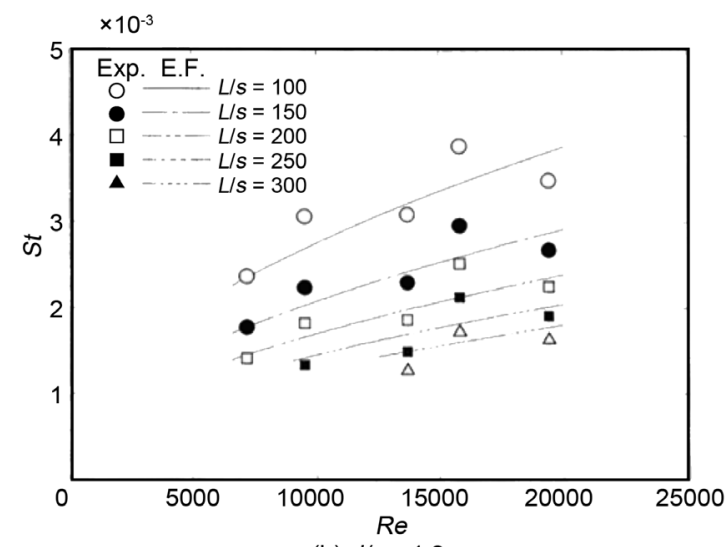

(b) $d / s=1.3$

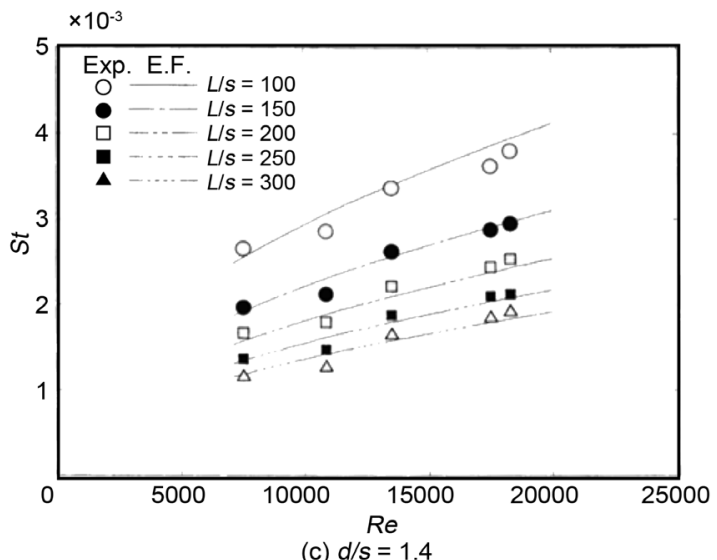

Figure 6. Strouhal number St against Reynolds number Re, in regular FFJN oscillation. Symbols and lines denote experiments and an empirical formula, respectively.

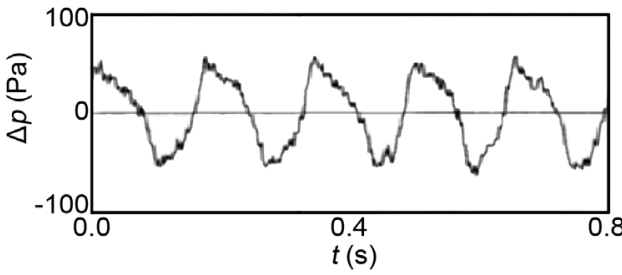

(a) Pressure difference $\Delta p$ by experiment

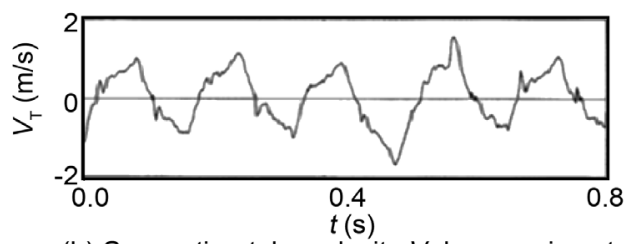

(b) Connecting-tube velocity $V_{\mathrm{T}}$ by experiment

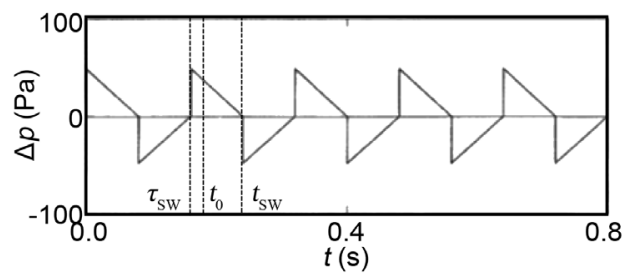

(c) Modeled pressure difference $\Delta p$ as a triangular wave

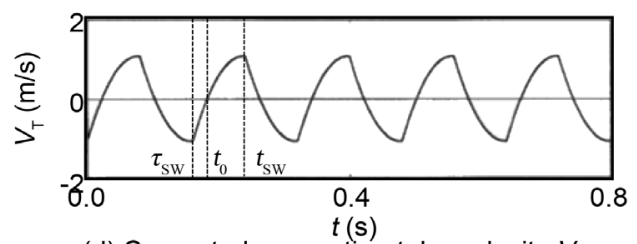

(d) Computed connecting-tube velocity $V_{T}$

Figure 7. Time histories of pressure difference $\Delta p$ between both connecting-tube ends and velocity $V_{\mathrm{T}}$ in a connecting tube, in regular FFJN oscillation at $L / s=100, d / s=1.2$ and $R e=13,000$.

measurements, we get Figure 7(b), which is the corresponding time history of the flow velocity $V_{\mathrm{T}}$ in the connecting tube obtained by the hot-wire anemometer (No. 13 in Figure 2). We can see that $V_{\mathrm{T}}$ is closely periodic, as well as $\Delta p$. Again, the periodicity is 
not rigorous due to high-frequency random fluctuations superimposed. While the wave form of $V_{\mathrm{T}}$ seems to be not sinusoidal but non-isosceles, it is rather different from the wave form of $\Delta p$ in Figure 7(a).

Now, we summarise all the experiments concerning the pressure difference $\Delta p$ from a quantitative point of view. Concerning the fluctuating period or the fluctuating frequency of $\Delta p$, we have already proposed Equation (3). Then, we next consider the fluctuating amplitude of $\Delta p$. To conclude, the pressure-difference-amplitude coefficient $C_{\triangle p \mathrm{AMP}}\left(\equiv \Delta p_{\mathrm{AMP}} /\left(1 / 2 \rho V_{\mathrm{PN}}{ }^{2}\right)\right)$, which is the normalised half value of the difference $\Delta p_{\text {AMP }}$ between the ensemble mean of the maximum $\Delta p$ and the ensemble mean of the minimum $\Delta p$, almost keeps a constant value of about 0.11 through all the present measurements, being independent of $L / s, d / s$ and $R e$.

At this stage, we attempt to purify these wave forms by a simple model which is the same as Funaki et al. [31]. This is because, the experimental raw data including random fluctuations like Figure 7(a) and Figure 7(b) are not suitable for further delicate discussion on the switching mechanism of the jet. At first, $\Delta p$ is simply modeled as a right-angled triangular wave, as shown in Figure 7 (c). Then, using the modeled $\Delta p$, we compute $V_{\mathrm{T}}$, as shown in Figure 7 (d). The computational procedure is as follows. As the compressibility is negligible through all the present experiments, the governing equations of motion can be described by

$$
\frac{d V_{\mathrm{T}}}{d t}=\frac{\Delta p}{\rho L}-\frac{\lambda}{d} \frac{V_{\mathrm{T}}\left|V_{\mathrm{T}}\right|}{2},
$$

where $V_{\mathrm{T}}$ denotes the flow velocity averaged over a cross section of the connecting tube to be exact. $\lambda$ is the resistance coefficient of pipe flow by Spriggs [32] and JSME [33], and are defined as follows. If $\operatorname{Re}_{\mathrm{CT}}\left(\equiv \rho V_{\mathrm{T}} s / \mu\right)<1900$, then

$$
\lambda=\frac{64}{R e_{\mathrm{CT}}} \text {. }
$$

If $1900 \leq R e_{\mathrm{CT}}<2900$, then

$$
\lambda=\frac{64}{R e_{\mathrm{CT}}}(1-\gamma)+\frac{0.3164}{R e_{\mathrm{CT}}^{1 / 4}} \gamma,
$$

where $\gamma=9.8 \times 10^{-4} R e_{\mathrm{CT}}-1.852$. And, if $2900 \leq R e_{\mathrm{CT}}<1,000,000$, then

$$
\lambda=\frac{0.3164}{R e_{\mathrm{CT}}^{1 / 4}} \text {. }
$$

We numerically solve Equation (4) by the fourth-order Runge-Kutta method. To confirm numerical accuracy, we compare several computations with different time steps. As a result, we can see that the wave form of the computed $V_{\mathrm{T}}$ in Figure 7 (d) is almost the same as the experiment at one in Figure 7(b), not only qualitatively but also quantitatively. Of course, the periodicity in Figure $7(\mathrm{~d})$ is rigorous, as the computed $V_{\mathrm{T}}$ does not include randomly-fluctuating components.

Now, we consider the physical background of the present approach. We assume that the jet switches, when the accumulation of the inflow into a jet-switching side wall from the connecting tube through a control port, and/or of the outflow from the opposite 
un-jet-switching side wall into the other control port and the connecting tube, reaches a certain value. As the accumulation, we examine the time integral $J_{\mathrm{P}}$ of mass flux, in addition to the time integral $J_{\mathrm{M}}$ of momentum flux for comparison. As mentioned in Section $1, J_{\mathrm{M}}$ is proposed by Funaki et al. [31]. $J_{\mathrm{P}}$ is the accumulated mass, which could be essentially regarded as the accumulated flow work by pressure to the fluid inside the re-circulation regions; strictly speaking, it is the product of the accumulated volume (or the quotient of the accumulated mass divided by $\rho$ ) and the pressure difference between a re-circulation region (Funaki et al. [19]) and the connecting-tube end (or the chamber) on the same side. So, we hereinafter refer to $J_{\mathrm{P}}$ as the accumulated flow work. Specifically speaking, the integrals $J_{\mathrm{P}}$ and $J_{\mathrm{M}}$ are defined as follows.

$$
\begin{gathered}
J_{\mathrm{P}} \equiv \frac{1}{S} \int_{t_{0}}^{t_{\mathrm{Sw}}} \rho Q w d t . \\
J_{\mathrm{M}} \equiv \frac{1}{S} \int_{t_{0}}^{t_{\mathrm{Sw}}} \rho V_{\mathrm{CP}} Q w d t .
\end{gathered}
$$

where $V_{\mathrm{CP}}$ denotes the flow velocity at the control port. In Equation (8) and Equation (9), a decaying factor $w$ is given by Equation (10).

$$
w \equiv \exp \left\{-\frac{\kappa\left(t_{\mathrm{SW}}-t\right) V_{\mathrm{PN}}}{s}\right\},
$$

where $\kappa$ denotes a damping constant. We should note that these integrals are amounts per unit span. To specify the integral interval in Equation (8) and Equation (9), Figure 7 (c) and Figure 7(d) show the definitions of $t_{0}$ and $t_{\mathrm{sw}}$. At $t=\tau_{\mathrm{sw}}, \Delta p$ jumps from zero to a positive value. This jump of $\Delta p$ corresponds to the jet's switching onto a side wall from the opposite side wall. On the other hand, $V_{\mathrm{T}}$ is still negative even at $t=\tau_{\mathrm{SW}}$, that is, the fluid flows in the connecting tube from the jet-switching side to the opposite un-jet-switching side. Thereafter, $\Delta p$ monotonically decreases with time $t$ toward zero. On the other hand, $V_{\mathrm{T}}$ monotonically increases with $t$ toward a certain positive value, crossing zero at $t=t_{0}$. Then, $V_{\mathrm{T}}$ becomes positive at $t>t_{0}$. In other words, the flow in the connecting tube is reversed at $t=t_{0}$ and afterwards continues to accelerate. Finally, at $t=t_{\mathrm{SW}}, \Delta p$ jumps from zero to a certain negative value, corresponding to the jet's switching from the side wall on the jet-switching side onto the opposite side wall. And, $V_{\mathrm{T}}$ begins to decelerate toward the next reverse of the connecting-tube flow from a certain positive value. In summary, there exists no reversed flow during the supposed integral interval in Equation (8) and Equation (9).

For convenience, all the integrals are usually normalised as follows.

$$
\begin{gathered}
J_{P}^{*} \equiv \frac{J_{\mathrm{P}}}{\rho s^{2}} \\
J_{M}^{*} \equiv \frac{J_{\mathrm{M}}}{\rho V_{\mathrm{PN}} s^{2}}
\end{gathered}
$$

Figure 8 shows the normalised integral $J_{P}^{*}$ (in Figure 8(a)), together with $J_{M}^{*}$ (in Figure 8(b)) plotted against the time constant $\kappa$, in a range of $L / s=100-300$ at $d / s=1.3$ and $R e=14,000$. While we suppose three governing parameters like $L / s, d / s$ and $R e$ 


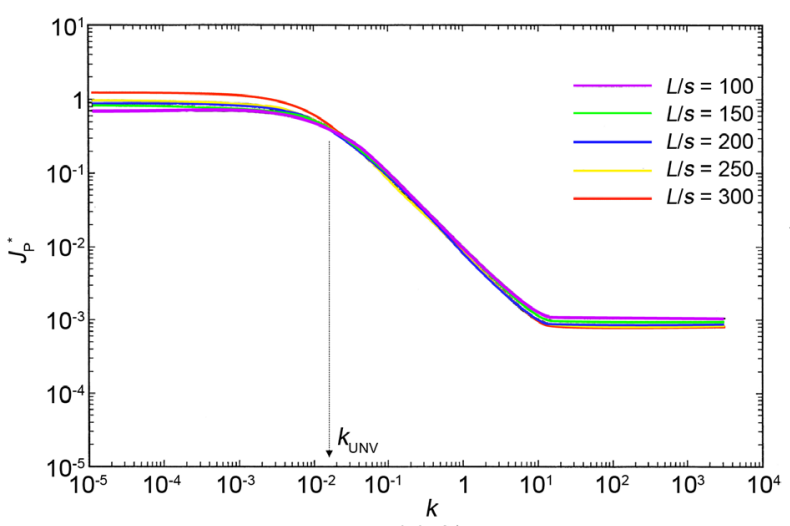

(a) $J_{p}^{*}$

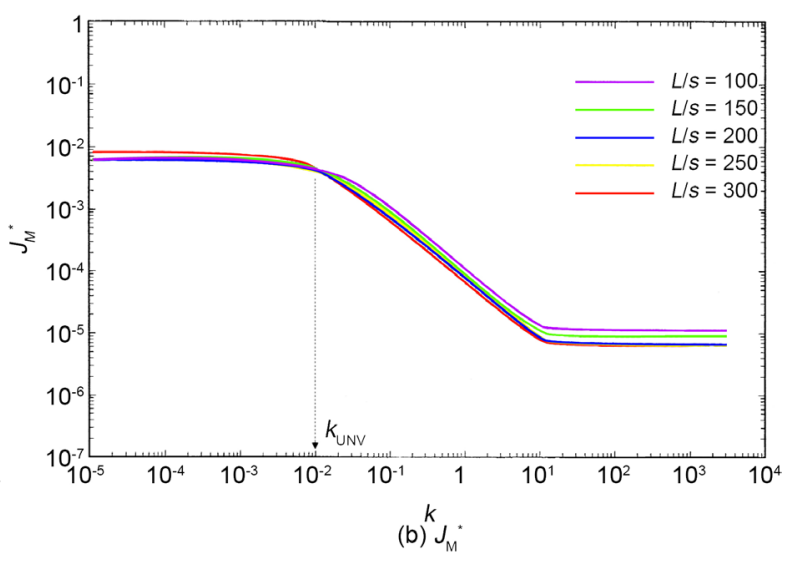

(b) ${ }^{k} J_{M}$

Figure 8. Normalised integrals $J_{P}^{*}$ and $J_{M}^{*}$ against time constant $\kappa$, at $L / s=100-300, d / s=1.3$ and $R e=14,000$.

in the present study, we have confirmed that $L / s$ is the most influential upon $S t$ among the three (also see Figure 6). So, we first examine the influence of $L / s$ prior to $d / s$ and $R e$.

At first, we see Figure 8(a). $J_{P}^{*}$ tends to decrease with increasing $\kappa$ at each $L / s$. As the decreasing manner of $J_{P}^{*}$ at each $L / s$ is not the same but depends upon $L / s$, the curve with a certain $L / s$ tends to cross the other curves with different $L / s$ s. Fortunately, we can find all the crosses in a narrow range of $\kappa \approx 0.01$. In other words, we can expect that $J_{P}^{*}$ is the universal number to determine the jet-oscillation period (or $S t$ ): that is, the jet switches when $J_{P}^{*}$ with $\kappa=\kappa_{\mathrm{UNV}}(=0.01)$ attains $\left(J_{P}^{*}\right)_{\mathrm{UNV}}(=0.2)$.

Second, we see Figure 8(b). $J_{M}^{*}$ tends to decrease with increasing $\kappa$ at each $L / s$, and the decreasing manner of $J_{M}^{*}$ depends upon $L / s$, as well as $J_{P}^{*}$. The curves with each $L / s$ tend to cross one another in a range of $\kappa \approx 0.006$. As the range is wider than that for $J_{P}^{*}$, all the curves in Figure 8(b) seems to focus less clearly than Figure 8(a). Thus, $J_{P}^{*}$ is considered to be more adequate than $J_{M}^{*}$, for the universal number to determine the jet-oscillation frequency. We have successfully found out the superiority of $J_{P}^{*}$ to $J_{M}^{*}$. As the reason for this finding, we should notice the present range of $S t$, which is much wider than that in Funaki et al. [31].

To conclude, concerning the influences of the other two governing parameters $d / s$ and $R e$ in addition to the influence of $L / s$, we summarise all the results in the experimental ranges such as $L / s=100-300, d / s=1.2-1.4$ and $R e=7000-20,000$ as follows. 1) $\kappa_{\mathrm{UNV}}$ is almost constant $(\approx 0.012)$ being independent of both $d / s$ and $\left.R e, 2\right)\left(J_{P}^{*}\right)_{\mathrm{UNV}}$ is almost independent of $d / s$. On the other hand, 3) we cannot ignore the influence of Re upon $\left(J_{P}^{*}\right)_{\mathrm{UNV}}$, which tends to monotonically decrease toward a constant value with increasing $R e$. This tendency is empirically given by

$$
\left(J_{P}^{*}\right)_{\mathrm{UNV}}=0.7 \exp \left(-8.0 \times 10^{-5} R e\right)+0.2 \text {, }
$$

on the basis of $\left(J_{P}^{*}\right)_{\mathrm{UNV}}$ for $L / s=100-300$ like Figure 8(a) at various values of $d / s$ and Re. Three constants in Equation (13) are derived using the least-squares method on the basis of all the results. This formula seems consistent, as the needed accumulated flow work for jet switching increases with decreasing $R e$.

As a result, the predicted St based on the empirical formula Equation (13) for 
$\left(J_{P}^{*}\right)_{\mathrm{UNV}}$ together with $\kappa=\kappa_{\mathrm{UNV}}(=0.012)$ assuming a triangular-wave pressure difference with $C_{\triangle p \mathrm{AMP}}=0.11$ shows good agreement with the experiment. In order to confirm the effectiveness of the empirical formula Equation (13), we have calculated the comparison between all the experiments and the corresponding predictions based on $\left(J_{P}^{*}\right)_{\mathrm{UNV}}$. As a result, the standard deviation of $S t$ is less than 0.0025 for all the present results. It should be remarked that both $C_{\Delta p \mathrm{AMP}}$ and $\kappa_{\mathrm{UNV}}$ are approximated to be constant without any dependences upon the three factors like $L / s, d / s$ and $R e$ in the prediction.

As will be revealed in the latter half of the present study, the inflow from one control port on the jet-reattaching wall is crucial for jet switching, while the outflow into the other control port on the opposite jet-un-reattaching wall is not crucial. At the present stage, although we do not have exact information to discuss the details of the jet switching mechanism, it seems acceptable that to weaken/destabilize the re-circulation region on the jet-reattaching wall could be a trigger of the jet's switching. In this context, the jet switching is possibly controlled by a certain accumulated amount from the control port, such as $J_{P}^{*}$ or $J_{M}^{*}$. It seems difficult to discuss the superiority $J_{P}^{*}$ or $J_{M}^{*}$ only from a theoretical point of view because the boundary of the re-circulation region is not exactly impermeable but complicated, rather soft, unsteady, turbulent and so on. However, as $J_{P}^{*}$ is one-order moment flux higher than $J_{M}^{*}, J_{P}^{*}$ more directly tends to affect the volume of the re-circulation region than $J_{M}^{*}$.

\subsection{Single-Port Control}

In the previous subsection, we have introduced $J_{P}^{*}$ as the universal number for jet switching. However, there exists one remaining question as to whether either or both of the inflows from the connecting tube into the jet-switching side wall and the outflow from the un-jet-switching side wall into the connecting tube are dominant. Another question is whether $\left(J_{P}^{*}\right)_{\mathrm{UNV}}$ is effective even in a quasi-steady situation at very low jet-oscillation frequency. So, in this subsection, in order to discuss the physics $J_{P}^{*}$, or the switching mechanism of the jet further, we conduct the single-port-control experiments. By some preliminary experiments, we have confirmed that the jet's switching is sensitive to the inflow, but not to the outflow. So, we will exclusively examine the effectiveness of $J_{P}^{*}$ concerning the inflow, below.

At first, we need to characterise the inflow from a quantitative point of view. Then, we assume a constant acceleration of the inflow or the connecting-tube flow, and define the flow-increment-rate coefficient $K$ as follows.

$$
K=\frac{d V_{\mathrm{T}} / d t b}{V_{\mathrm{PN}}{ }^{2}}
$$

$K$ means a normalised acceleration of fluid in the tube. From a theoretical point of view, $K$ or the acceleration $d V_{\mathrm{T}} / d t$ ought to be constant, but vary with time $t$. However, as seen in Figures $7(\mathrm{~b})-(\mathrm{d}), V_{\mathrm{T}}$ could increase approximately with a constant acceleration from the reversed time $t_{0}$ to the jet-switching time $t_{\mathrm{sw}}$. In other words, we could suppose the connecting-tube flow and the inflow continue to accelerate linearly until 
the instant when the jet switches. Under this situation, $K$ becomes an appropriate parameter.

Next, we estimate the range of $K$ in the actual regular oscillation of the ordinary FFJN with two control ports. Figure 9 shows $K$ plotted against $R e$, in the regular oscillation at $L / s=100-300$ and $R e=5000-25,000$. Figure 9(a) and Figure 9(b) denote the results at $d / s=1.2$ and 1.4, respectively. More specifically, Figure 9 is based on the computed $V_{\mathrm{T}}$ 's like Figure $7(\mathrm{~d})$. We should note that $K$ is the time-mean value from $t_{0}$ to $t_{\mathrm{sW}}$, because $K$ depends upon $t$ in a strict sense. We can confirm the dependence of $K$ upon $L / s, d / s$ and $R e$. The actual range of $K$ in the regular oscillation varies from $1 \times$ $10^{-4}$ to $5 \times 10^{-4}$. So, we next conduct the single-port-control experiments in a range of $K$ from $1 \times 10^{-4}$ to $3.5 \times 10^{-4}$, keeping a constant acceleration of the inflow as closely as possible. To be exact, although $K$ in the regular oscillation is not the same as that in the single-port-control experiments, the order of $K$ is the same.

Figure 10 and Figure 11 show typical examples in the single-port-control experiment, namely, the time histories of two velocities $V_{\mathrm{T}}$ and $V_{\mathrm{EX}}$ and a pressure $p_{\mathrm{TE}}$ where $V_{\mathrm{EX}}$ and $p_{\mathrm{TE}}$ denote the flow velocity near one side wall at the FFJN's exit and the pressure at the connecting-tube end adjacent to the chamber and the control port. Figure 10 denotes the results at $K=1 \times 10^{-4}$ and $R e=8800$, and Figure 11 denotes those $K=$ $3.5 \times 10^{-4}$ and $R e=8800$. In each figure, figures (a), (b) and (c) represent $V_{\mathrm{T}}, V_{\mathrm{EX}}$ and $p_{\text {TE }}$, respectively.

At first, we see Figure 10. In Figure 10(a), $V_{\mathrm{T}}$ starts to increase from zero with time $t$ at $t=t_{0}(=0.6 \mathrm{~s})$. We should note that the instant at $t=0$ merely represents the time when each measurement starts. The increasing manner is not strictly linear, but almost linear with a constant acceleration during the duration $t=0.6-1.1 \mathrm{~s}$. At $t=t_{\mathrm{SW}}$, the jet from the primary nozzle switches from the beforehand-jet-attached side to the opposite afterward-jet-attached side. In order to determine $t_{\mathrm{SW}}$, this switching is preliminarily observed by flow visualisation using smoke together with simultaneous measurements of $V_{\mathrm{T}}, V_{\mathrm{EX}}, p_{\mathrm{TE}}$ and so on. Actually, corresponding to this jet switch at $t=t_{\mathrm{SW}}, V_{\mathrm{EX}}$ in Figure 10(b) and $p_{\mathrm{TE}}$ in Figure 10(c) step down and up toward constant values at the

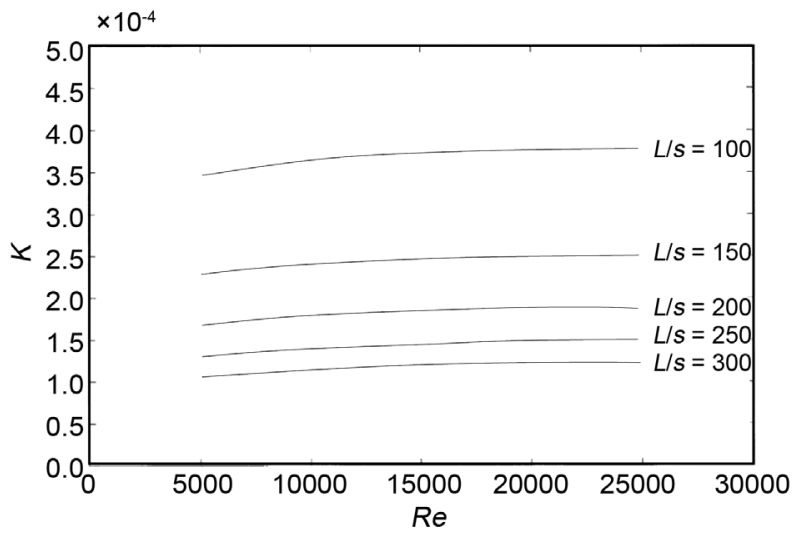

(a) $d / s=1.2$

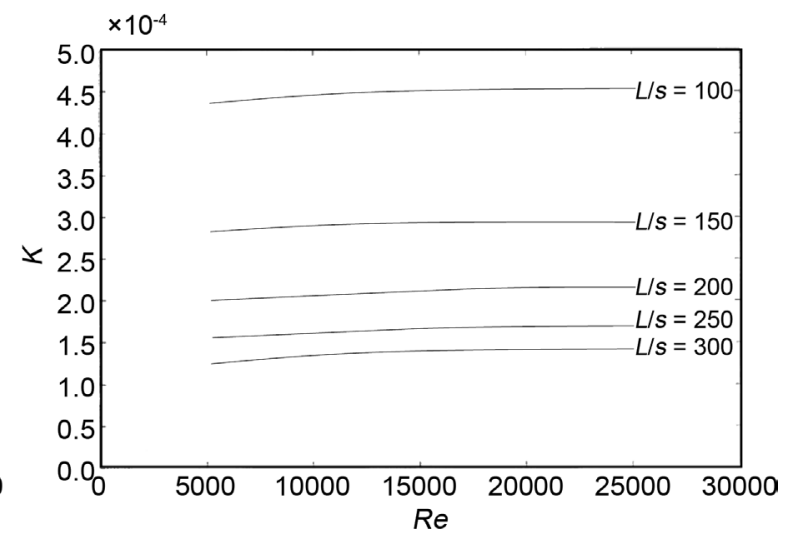

(b) $d / s=1.4$

Figure 9. Flow-increment-rate coefficient $K$ against $R e$, in regular FFJN oscillation. 


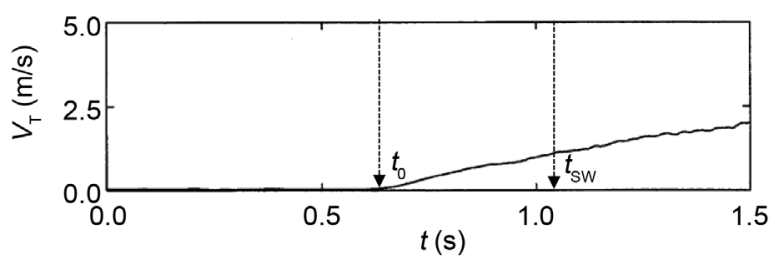

(a) Time history of flow velocity $V_{\mathrm{T}}$ in thr connecting tube.

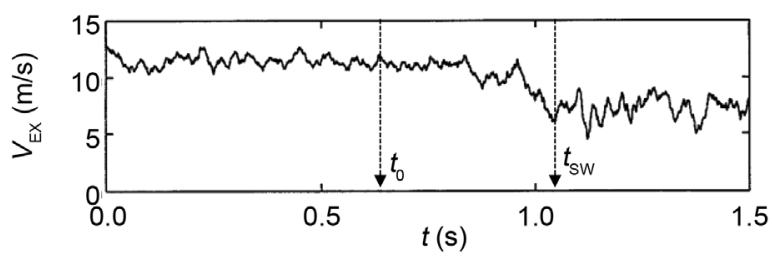

(b) Time history of flow velocity $V_{E X}$ at a FFJN exit near one side wall.

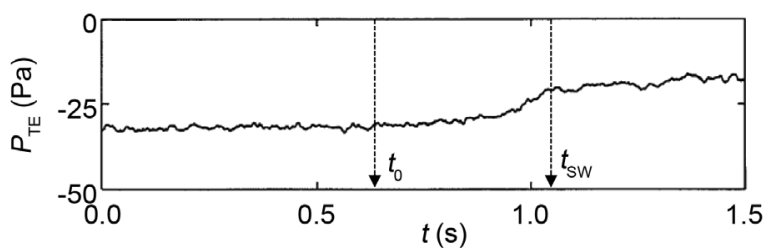

(c) Time history of pressure $P_{\mathrm{TE}}$ at the connecting tube end.

(a) $K=1.0 \times 10^{-4}$

Figure 10. Time histories of velocities and pressure in single-port control at $K=1.0 \times 10^{-4}$ and $R e=8800$.

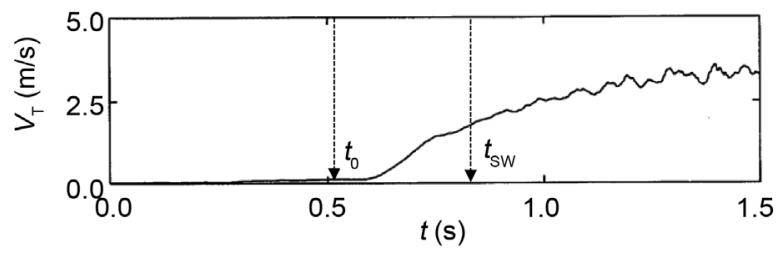

(a) Time history of flow velocity $V_{T}$ in thr connecting tube.

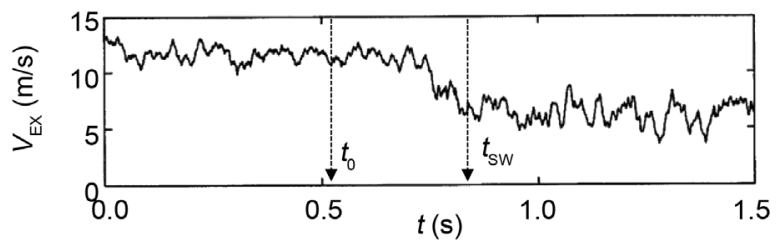

(b) Time history of flow velocity $V_{\text {EX }}$ at a FFJN exit near one side wall.

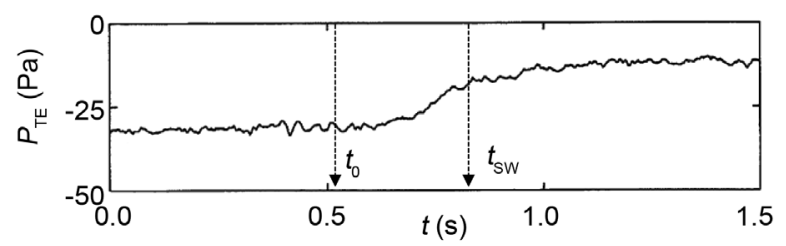

(c) Time history of pressure $P_{\mathrm{TE}}$ at the connecting tube end.

(B) $K=3.5 \times 10^{-4}$

Figure 11. Time histories of velocities and pressure in single-port control at $K=3.5 \times 10^{-4}$ and $R e=8800$. 
same time, respectively. Then, we can determine $K$ or $d V_{\mathrm{T}} / d t$ from Figure 10 . To be exact, $d V_{\mathrm{T}} / d t$ is time-mean which is obtained by such three data as $t_{0}, t_{\mathrm{SW}}$ and the $V_{\mathrm{T}}$ at $t=t_{\mathrm{SW}}$. Of course, the above features can be seen in Figure 11 as well as Figure 10.

Figure 12 summarises all the results in the single-port-control: measured $J_{P}^{*}$ 's in the single-port-control experiments are plotted against $R e$ in a range of $R e=8000$ 20,000. Figure 12(a) denotes at $d / s=1.3$ and various values of $K$. And, Figure 12(b) denotes at $K=3 \times 10^{-4}$ and at various values of $d / s$. Each plot represents the ensemble mean over five trials in the single-port-control experiments. And, a solid line represents proposed empirical formula Equation (13) for $\left(J_{P}^{*}\right)_{\mathrm{UNV}}$ based on the regular oscillation using the ordinary FFJN with two control ports in connection. We can see good agreement of the single-port control with the empirical formula for the regular oscillaton. This agreement suggests that $J_{P}^{*}$ or the accumulated flow work of not the outflow but the inflow from the connecting tube to the FFJN inside could be a key parameter for jet switching to explain the oscillation mechanism of the FFJN, in addition to the validity of $J_{P}^{*}$ in practical aspects to estimate the jet frequency of the FFJN.

\section{Conclusion}

In order to reveal the oscillation mechanism of a flip-flop jet nozzle (FFJN) with a connecting tube, we have carried out the measurements of pressures and velocities in the connecting tube and inside the FFJN specially focusing on the jet-oscillation frequency $f$, varying: 1) the diameter $d$ of the connecting tube; 2) the length $L$ of the connecting tube and 3) the jet velocity $V_{\mathrm{PN}}$ from a primary-nozzle exit. Obtained results are as follows. We have proposed an empirical formula to determine $f$, and confirmed its validity. Then, to consider $f$ more generally, we assume that the jet switches when a time integral reaches a certain value. At first, as the time integral, we have introduced the accumulated flow work $J_{P}^{*}$ of pressure through the connecting tube into the jet- reattaching wall from the opposite jet-un-reattaching wall. Under this assumption, we have conducted computations, and shown the effectiveness of $J_{\mathrm{P}}$ to determine $f$. Second, to discuss the physics of $J_{\mathrm{P}}$ further, we have conducted another experiment in single- port control, instead of the experiment in regular jet oscillation using the ordinary nozzle

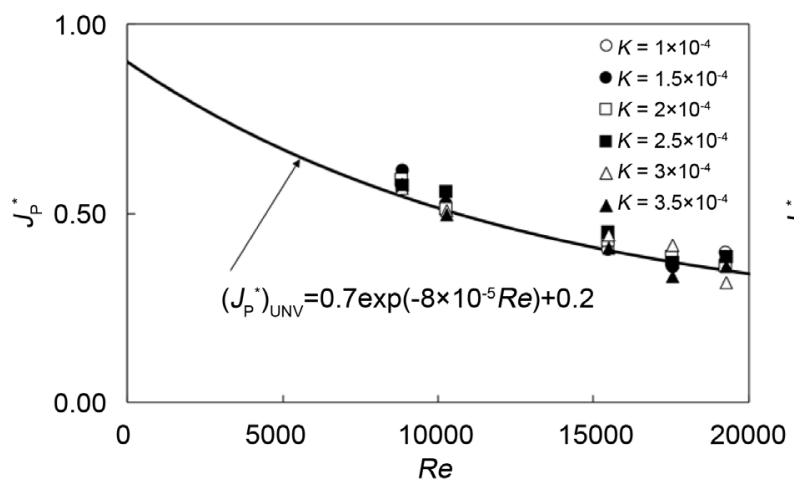

(a) $d / s=1.3$

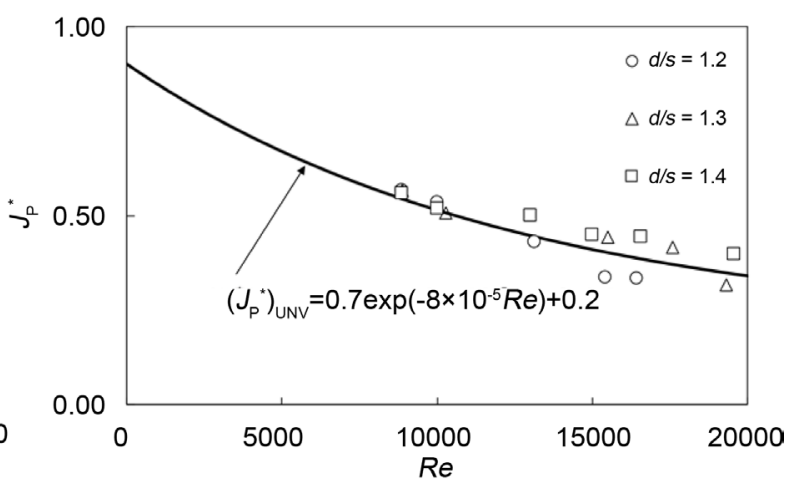

(b) $K=3 \times 10^{-4}$

Figure 12. Time histories of velocities and pressure in single-port control at $K=1 \times 10^{-4}$ and $\operatorname{Re}=20,000$. 
with two control ports in connection. As the result, we have confirmed good agreement between the single-port control and the regular jet oscillation. This agreement suggests that $J_{\mathrm{p}}$ from the connecting tube to the FFJN inside can be a key parameter to explain the jet's switching, in addition to the validity of $J_{\mathrm{P}}$ in practical aspects to estimate the dominant jet frequency of the FFJN.

\section{References}

[1] Levin, S.G. and Manion, F.M. (1962) Jet Attachment Distance as a Function of Adjacent Wall Offset and Angle. Fluid Amplification, 5, 20-22.

[2] Ozaki, S. and Hara, Y. (1967) Introduction to Fluid Logic. Nikkan Kogyo Shimbun Ltd., Tokyo. (In Japanese)

[3] Perry, C.C. (1967) Two-Dimensional Jet Attachment. In: Brown, F.T., Ed., Advances in Fluidics, ASME, New York, 205-217.

[4] Epstein, M. (1971) Theoretical Investigation of the Switching Mechanism in a Bistable Wall Attachment Fluid Amplifier. ASME Journal of Fluids Engineering, 93, 55-62. http://dx.doi.org/10.1115/1.3425182

[5] Drzewiecki, T.M. and Goto, J.M. (1973) An Analytical Model for the Response of Flueric Wall Attachment Amplifier. Fluidics Quarterly, 5, 43-65.

[6] Wilson, M.P., Coogan, C.H. and Southall, K. (1970) Experimental Investigation of a Fluidic Volume Flowmeter. ASME Journal of Fluids Engineering, 92, 139-142. http://dx.doi.org/10.1115/1.3424922

[7] Tippetts, J.R., Ng, H.K. and Royle, J.K. (1973) A Fluidic Flowmeter. Automatica, 9, 35-45. http://dx.doi.org/10.1016/0005-1098(73)90010-1

[8] Viets, H. (1975) Flip-Flop Jet Nozzle. AIAA Journal, 13, 1375-1379. http://dx.doi.org/10.2514/3.60550

[9] Morris, G.J., Jurewicz, J.T. and Palmer, G.M. (1992) Gas-Solid Flow in a Fluidically Oscillating Jet. ASME Journal of Fluids Engineering, 114, 362-366. http://dx.doi.org/10.1115/1.2910038

[10] Raman, G., Hailye, M. and Rice, E.J. (1993) Flip-Flop Jet Nozzle Extended to Supersonic Flows. AIAA Journal, 31, 1028-1035. http://dx.doi.org/10.2514/3.11725

[11] Raman, G. and Rice, E.J. (1994) Development of Phased Twin Flip-Flop Jets. ASME Journal of Vibration and Acoustics, 116, 263-268. http://dx.doi.org/10.1115/1.2930423

[12] Raman, G., Rice, E.J. and Cornelius, D.M. (1994) Evaluation of Flip-Flop Jet Nozzle for Use as Practical Excitation Devices. ASME Journal of Fluids Engineering, 116, 508-515. http://dx.doi.org/10.1115/1.2910306

[13] Raman, G. (1997) Using Controlled Unsteady Fluid Mass Addition to Enhance Jet Mixing. AIA A Journal, 35, 647-656. http://dx.doi.org/10.2514/2.185

[14] Camci, C. and Herr, F. (2002) Forced Convection Heat Transfer Enhancement Using a SelfOscillating Impinging Planar Jet. ASME Journal of Heat Transfer, 124, 770-782. http://dx.doi.org/10.1115/1.1471521

[15] Hung, C.I., Wang, K.C. and Chyou, C.K. (2005) Design and Flow Simulation of a New Micromixer. JSME International Journal Series B, 48, 17-24. http://dx.doi.org/10.1299/jsmeb.48.17

[16] Gregory, J.W., Sullivan, J.P. and Raghu, S. (2005) Visualization of Jet Mixing in a Fluidic Oscillator. Journal of Visualization, 8, 169-176. http://dx.doi.org/10.1007/BF03181660 
[17] Gregory, J.W., Sullivan, J.P., Raman, G. and Raghu, S. (2007) Characterization of the Microfluidic Oscillator. AIAA Journal, 45, 568-576. http://dx.doi.org/10.2514/1.26127

[18] Yang, J.T., Chen, C.K., Tsai, K.J., Lin, W.Z. and Sheen, H.J. (2007) A Novel Fluidic Oscillator Incorporating Step-Shaped Attachment Walls. Sensors and Actuators A: Physical, 135, 476-483. http://dx.doi.org/10.1016/j.sna.2006.09.016

[19] Funaki, J., Matsuda, Y., Inoue, T., Tanigawa, H. and Hirata, K. (2007) UVP Measurements on Periodic Flow in a Flip-Flop Jet Nozzle. Journal of Fluid Science and Technology, 73, 133-138.

[20] Cerretelli, C. and Kirtley, K. (2009) Boundary Layer Separation Control with Fluidic Oscillators. ASME Journal of Turbomachinery, 131, Article ID: 041001. http://dx.doi.org/10.1115/1.3066242

[21] Tesar, V., Zhong, S. and Rasheed, F. (2013) New Fluidic-Oscillator Concept for Flow-Separation Control. AIAA Journal, 51, 397-405. http://dx.doi.org/10.2514/1.J051791

[22] Metka, M. and Gregory, J.W. (2015) Drag Reduction on the 25-deg Ahmed Model Using Fluidic Oscillators. ASME Journal of Fluids Engineering, 137, Article ID: 051108. http://dx.doi.org/10.1115/1.4029535

[23] Yamasaki, H., Takahashi, A. and Honda, S. (1988) A New Fluidic Oscillator for Flow Measurement. Proceedings of FLUCOM, Sheffield, 1988, 16-20.

[24] Shakouchi, T. (1989) A New Fluidic Oscillator, Flowmeter, without Control Port and Feedback Loop. ASME Journal of Dynamic Systems, Measurement, and Control, 111, 535539. http://dx.doi.org/10.1115/1.3153085

[25] Mi, J., Nathan, G.J. and Luxton, R.E. (2001) Mixing Characteristics of a Flapping Jet from a Self-Exciting Nozzle. Flow, Turbulence and Combustion, 67, 1-23. http://dx.doi.org/10.1023/A:1013544019463

[26] Priestman, G.H. and Boucher, R.F. (2005) The Biased Laminar By-Pass Fluidic Flowmeter. ASME Journal of Fluids Engineering, 127, 1199-1204. http://dx.doi.org/10.1115/1.2060729

[27] Priestman, G.H. and Boucher, R.F. (2006) Smart Fluidic Meters for Simultaneous Measurement of Fluid Flowrate, Reynolds Number, Density and Viscosity. Journal of Chemical Engineering of Japan, 39, 383-393. http://dx.doi.org/10.1252/jcej.39.383

[28] Huang, R.F. and Chang, K.T. (2007) Evolution and Turbulence Properties of Self-Sustained Transversely Oscillating Flow Induced by Fluidic Oscillator. ASME Journal of Fluids Engineering, 129, 1038-1047. http://dx.doi.org/10.1115/1.2746905

[29] Hirata, K., Matoba, N., Naruse, T., Haneda, Y. and Funaki, J. (2009) On the Stable-Oscillation Domain of a Simple Fluidic Oscillator. Journal of Fluid Science and Technology, 4, 623-635. http://dx.doi.org/10.1299/jfst.4.623

[30] Hirata, K., Inoue, T., Haneda, Y., Miyashita, N., Tanigawa, H. and Funaki, J. (2011) On Dominant Oscillation Frequency of a Simplified Fluidic Oscillator. Journal of Fluid Science and Technology, 6, 534-547. http://dx.doi.org/10.1299/jfst.6.534

[31] Funaki, J., Mizuno, G., Kondo, M. and Hirata, K. (1999) Oscillation Mechanism of a FlipFlop Jet Nozzle Based on the Flow Which Flows through a Connecting Tube. Transactions of the JSME, Series B, 65, 928-933. (In Japanese) http://dx.doi.org/10.1299/kikaib.65.928

[32] Spriggs, H.D. (1973) Comments on Transition from Laminar to Turbulent Flow. Industrial and Engineering Chemistry Fundamentals, 12, 286-290.

http://dx.doi.org/10.1021/i160047a004

[33] The Japan Society of Mechanical Engineers, Ed. (1979) JSME Data Handbook: Hydronic Losses on Pipes and Ducts, JSME, Tokyo, 22-26. (In Japanese) 


\section{Nomenclature}

A: $\quad$ aspect ratio of a primary-nozzle throat, $\equiv S / s$

b: $\quad$ breadth of a control port $(\mathrm{m})$

$C_{\Delta p \mathrm{AMP}}: \quad$ coefficient of pressure-difference amplitude $\equiv \Delta p_{\mathrm{AMP}} /\left(1 / 2 \rho V_{\mathrm{PN}}^{2}\right)$

$d: \quad$ (inner) diameter of a connecting tube $(\mathrm{m})$

f. frequency $(\mathrm{Hz})$

$G_{\text {sw }}: \quad$ gap between side walls (m)

$J_{\mathrm{M}}: \quad$ time integral of momentum flow per unit span $(\mathrm{kg} / \mathrm{s})$

$J_{\mathrm{p}}: \quad$ time integral of mass flow per unit span $(\mathrm{kg} / \mathrm{m})$

$K: \quad$ flow-increment-rate coefficient

$L: \quad$ length of a connecting tube $(\mathrm{m})$

$L_{\mathrm{SW}}: \quad$ streamwise length of a side wall $(\mathrm{m})$

p: $\quad$ pressure $(\mathrm{Pa})$

$\Delta p: \quad$ pressure difference between two connecting-tube ends $(\mathrm{Pa})$

$\Delta p_{\text {AMP }}: \quad$ (half) amplitude of $\Delta p(\mathrm{~Pa})$

Q. $\quad$ (volumetric) flow rate $\left(\mathrm{m}^{3} / \mathrm{s}\right)$

Re: Reynolds number, $\equiv \rho V_{\mathrm{PN}} s / \mu$

$R e_{\mathrm{CT}}: \quad$ connecting-tube Reynolds number, $\equiv \rho V_{\mathrm{T}} d / \mu$

$S: \quad \operatorname{span}(\mathrm{m})$

s. $\quad$ spacing of a primary-nozzle throat $(\mathrm{m})$

St. $\quad$ Strouhal number, $\equiv f \mathcal{S} / V_{\mathrm{PN}}$

t. time (s)

$V: \quad$ flow velocity $(\mathrm{m} / \mathrm{s})$

$w$ decaying factor

$\lambda: \quad$ friction coefficient of pipe

$\kappa$ damping constant

$\mu: \quad$ viscosity of fluid $(\mathrm{Pa} \cdot \mathrm{s})$

$\rho: \quad$ density of fluid $\left(\mathrm{kg} / \mathrm{m}^{3}\right)$

$\tau_{\mathrm{sw}}: \quad$ time at former jet's switching (s) 
Submit or recommend next manuscript to SCIRP and we will provide best service for you:

Accepting pre-submission inquiries through Email, Facebook, LinkedIn, Twitter, etc. A wide selection of journals (inclusive of 9 subjects, more than 200 journals)

Providing 24-hour high-quality service

User-friendly online submission system

Fair and swift peer-review system

Efficient typesetting and proofreading procedure

Display of the result of downloads and visits, as well as the number of cited articles

Maximum dissemination of your research work

Submit your manuscript at: http://papersubmission.scirp.org/

Or contact jfcmv@scirp.org 